\section{Molecular analysis of}

\section{immunoglobulins related to salmonella typhi in pediatric patients}

\author{
Farah Qudsia1, Muhammad Rehan² and Samreen Riaz ${ }^{3 *}$ \\ ${ }^{1}$ Department of Microbiology and Molecular Genetics, University of the Punjab, Lahore, Pakistan \\ ${ }^{2}$ Research Officer, loT Research and Innovation Lab, Al-Khwarizmi Institute of Computer Sciences, \\ University of Engineering and Technology, Lahore, Pakistan \\ ${ }^{3}$ Assistant Professor, Department of Microbiology and Molecular Genetics, University of the Punjab, \\ Lahore, Pakistan
}

\section{Abstract}

Typhoid fever is a systemic infection caused by Salmonella enterica serotype typhi. It is of major concern in tropical regions of the world. Highest episodes of typhoid fever occur in Asia i.e. $93 \%$. Early diagnosis of the disease is mandatory to lower the mortality rate associated with it as well as to prevent the emergence of antimicrobial drug resistance by Salmonella typhi. Research work was conducted in Immunology Department of the Children's Hospital, Lahore for the period of one year including a total of 60 patients suspected of having typhoid fever. Serum samples of these patients were tested for Typhidot IgG and IgM antibodies as well as for the antibodies against TO and TH antigens using Widal test. Of the total 60 patients, $10(16.7 \%)$ were positive for both Typhidot IgG and IgM, $16(26.7 \%)$ were positive for Typhidot IgM, $3(5 \%)$ were Positive for Typhidot IgG and 31 (51.66\%) were negative for both Typhidot IgG and IgM. Reading the results of Widal test, $8(13.33 \%)$ were positive for Widal TO and TH antigens, $3(5 \%)$ were positive for Widal TO antigen, $19(31.7 \%)$ were positive for Widal TH antigen and $30(50 \%)$ were negative for Widal $\mathrm{TO}$ and $\mathrm{TH}$ antigens. IgM is positive at the early stage of acute typhoid fever, IgM along with IgG positive means the middle stage of acute illness. The detection of only IgG cannot discriminate between acute and convalescent phases as it can stay in the serum for at least 2 years or more. The Typhidot test is much helpful for the rapid diagnosis of typhoid fever as compared to Widal test which is still being used in some set ups in poor countries, although has become mostly obsolete. By testing the rise of $\operatorname{lgM}$ and $\lg G$ antibodies against Salmonella typhi, we can detect the infection at early and late stages, respectively.

\section{More Information}

*Address for Correspondence: Samreen Riaz, Assistant Professor, Department of Microbiology and Molecular Genetics, University of the Punjab, Lahore, Pakistan,

Email: samreen.mmg@pu.edu.pk

\section{Submitted: 11 April 2020}

Approved: 23 April 2020

Published: 24 April 2020

How to cite this article: Qudsia F, Rehan M, Riaz S. Molecular analysis of immunoglobulins related to salmonella typhi in pediatric patients. Arch Pathol Clin Res. 2020; 4: 005-010. DOI: 10.29328/journal.apcr.1001017

Copyright: (c) 2020 Qudsia F, et al. This is an open access article distributed under the Creative Commons Attribution License, which permits unrestricted use, distribution, and reproduction in any medium, provided the original work is properly cited.

Keywords: Febril illness; Typhidot; Widal; Immunoglobulin G; Immunoglobulin M

Check for updates

OPEN ACCESS

\section{Introduction}

Salmonella enterica infections are a unifying common cause of bloodstream infection in developing countries. In these areas, it is related to a high case fatality quantitative relation [1]. Typhoid, caused by the Gram-negative bacterium Salmonella enterica serovar Typhi, could be a major explanation for community-acquired bacteremia and death worldwide [2]. Various clinical presentations are associated with it which also overlap many other acute febrile illnesses [3]. Noticeable cases of enteric fever caused by Salmonella enterica serovars Typhi and Paratyphi are routinely reported which have high impact in toddlers and youngsters [4]. It is a common childhood problem in both urban and rural regions in tropical countries. Especially Asia, Africa, Eastern Europe, and Latin America are at high risk [3]. Asia is mostly affected by it contributing $93 \%$ of the global episodes. The major causative factors are poor hygiene, poor sanitation and sewerage system and over population [5]. The predominant mode of transmission was believed to be the direct fecal-oral route but recent proof suggests that indirect environmental transmission may additionally contribute in spreading the disease [6]. It is still an increasing problem in under developed countries like Pakistan [7]. Salmonalla enterica has initially acquired antimicrobial resistance against the traditional first-line drugs like chloramphenicol, ampicillin, and trimethoprim-sulfamethoxazole [1]. The major cause of the emergence of antibiotic resistance is late treatment of typhoid fever due to incorrect diagnostic assay results and their interpretation. The arrival of a speedy check for identification of typhoid has returned as a blessing for clinicians and patients [8]. Typhoid fever cannot be diagnosed by the physician on the basis of clinical features as they are very similar in common infections like malaria, dengue, typhoid, leptospirosis and scrub typhus [9]. An early and correct identification is important for a prompt and 
effective treatment [10]. A simple, reliable, affordable, and speedy diagnostic assay has been a long-felt requisite of the cliniciansc [11]. Form always for its identification are blood culture and Widal test. The gold standard for Typhoid fever is blood culture but is time consuming [12]. Also, the results of the blood culture are affected by prior administration of antibiotics. Widal test gives positive results after increasing titers and this is often not helpful for early medical care [13]. Typhidot is a speedy serological test that is now commercially accessible and reliable in identification of typhoid fever even with restricted resources [14]. It is a dot enzyme immunoassay (EIA) that identifies the immunoglobulins IgM and IgG against the specific 50KD outer membrane protein (OMP) of the Salmonella typhi. Typhidot has been used as the sole tool to diagnose typhoid fever by general practitioners and consultants [7]. It is reasonable and a reliable diagnostic test for identification of typhoid in resource-limited setting among patients with a very high index of suspicion of infectious disease when blood culture is not accessible or possible [8].

\section{Aims and objectives}

Keeping in view the above literature, aims and objectives of research work were to determine the Typhidot IgG and IgM antibodies as well as the titer antibodies against TO and $\mathrm{TH}$ antigens using Widal test in patients suspected of having typhoid fever.

\section{Inclusion criteria}

Patients of either sex with age range of 1 year to 14 years.

\section{Exclusion criteria}

Patients with fever with some definite focus of infection.

\section{Materials and methods}

Duringa period of one year, all patients having a clinical suspicion of typhoid fever, reporting to the Department of Immunology, Children's Hospital Lahore, were included in this study. The patients were children between ages of 1 to 14 years and of both sexes with clinical features suggestive of typhoid fever.

\section{Data collection and clinical history}

For data collection and clinical history, a proforma was developed. The parameters taken into consideration for recording the clinical history were fever, weakness, anorexia, abnormal bowel symptoms like diarrhea or constipation, abdominal pain, nausea, vomiting, cough, chest discomfort, sore throat and coated tongue.

\section{Sample collection}

5c.c. of blood was collected aseptically from the anti-cubical vein of each patient. The blood samples were centrifuged and the serum separated was divided into two parts.

\section{Tests performed}

Typhidot and Widal tests were performed on the serum sample for the serological diagnosis of typhoid fever. The kit used for Typhidot tes was "Malaysian Biodiagnostic Research SDN BHD, Singapore, Malaysia". Whereas Widal test was performed using "Sanofi qualitative agglutination test kits (Bio-Rad)". The tests were performed according to manufacturer's instruction.

\section{Protocol of typhidot test}

To perform Typhidot test the reaction tray was divided into two columns. One was marked as "M" and one column as "G". Predotted antigen strips were removed using the forceps and placed into the appropriate reaction wells. $250 \mu$ l of the sample diluent was added into the appropriate " $M$ " and "G" reaction wells. Tray was shaked gently to allow strips to be thoroughly wet. $2.5 \mu \mathrm{l}$ of either control or test serum was added to the appropriate "M" or "G" reaction wells to achieve a final serum dilution of 1:100. The solution was aspirated gently to mix. The tray having solutions was incubated on a rocker platform for 20 minutes. The antibody solution was aspirated into a discard jar containing disinfectant. $250 \mu \mathrm{l}$ of prepared washing buffer was added into each well and washed $3 \mathrm{X}$ for five minutes. $250 \mu \mathrm{l}$ of prediluted Anti-Human IgM was added to the " $M$ " well and $250 \mu \mathrm{l}$ of prediluted AntiHuman IgG into the "G" well using sterile micropipette tips. The tray was covered with a cover for 15 minutes at room temperature on a rocker platform. The $2^{\text {nd }}$ antibody solution was aspirated and washed 3X for 5 minutes. $250 \mu$ of the color development solution was added into each well. The tray was covered and incubated on the rocker platform. Fifteen minutes were allowed for colour development. The reaction was stopped by aspirating the solution and briefly rinsing the strip in distilled water (3X). Strips were placed on the filer paper to dry and then results were interpreted as:

Negative result: Antigen coated strip was as blank as it was before starting the reaction.

Positive result: Antigen coated strip was showing two blue dots on it. A positive IgM was inferred as acute typhoidal illness, while IgM and IgG positive were meant for acute typhoidal illness in middle stage of infection and IgG positive was inferred as chronic carrier or previous infection or reinfection.

\section{Protocol for widal test}

To perform Widal test, the test reagents and sample were brought to the room temperature. Antigen vials were re-suspended gently. Clear glass slides were taken. For each specimen to be tested, drops of $80,40,10$ and $5 \mu \mathrm{l}$ of the patient's serum were placed into separate circles of slides (for making the dilution of 1/20,1/40,1/80,1/160 and $1 / 320$, respectively). A $40 \mu \mathrm{l}$ drop of the appropriate antigen 
suspension $(\mathrm{O}$ or $\mathrm{H})$ was added next to the drops of serum. Slide was rocked gently by hand for 1 minute. Results were interpreted as following:

Negative results: Smooth suspension with no visible agglutination.

Positive result: Any degree of agglutination visible microscopically. Tire of $1 / 160$ or above for $\mathrm{TO}$ or $\mathrm{TH}$ antigens was considered as positive for typhoid fever.

\section{Results}

The study included a total of 60 patients both males and females, suspected of having typhoid fever, who reported to the Children's Hospital Lahore and were referred to Immunology Department for serological diagnosis of typhoid fever.

In the study population $(n=60)$ of pediatric patients suspected of having typhoid fever 38 (63\%) were males while $22(37 \%)$ were females with a male: female ratio of 1.7:1 (Figure 1a).

Of the total 60 patients, $10(16.66 \%)$ were positive for both Typhidot IgG and IgM, 16 (26.66\%) were positive for Typhidot lgM, 3 (5\%) were positive for Typhidot IgG and
31 (51.66\%) were negative for both Typhidot IgG and IgM (Figure 1b).

A total of 10 patients with Typhidot positive results were identified, in these cases, equal number of males and females was affected. In 16 Typhidot IgM positive patients, $11(68.75 \%)$ were males and 5 (31.25\%) were females. In the Typhidot IgG positive patients all were females (100\%). Among rest of the 31 Typhidot lgG and IgM negative patients $23(74.19 \%)$ were males and $8(25.80 \%)$ were females. Out of total patient population $(n=60)$, based on duration of fever, $11(18.33 \%)$ patients were having fever for $<7$ days, 23 (38.33\%) were having fever for 7-14 days and 26 (43.33\%) were having fever for $>14$ days (Figure 2a).

Amongst patients having fever for $<7$ days 2 (3.33\%) were positive for both Typhidot $\lg G$ and $\lg M$, no patient $(0 \%)$ was positive for either Typhidot IgG or Typhidot IgM alone while $9(15 \%)$ were negative for both Typhidot IgG and IgM. Amongst patients having fever for 7-14 days, 4 (6.66\%) were positive for both Typhidot IgG and IgM, 7 (11.66\%) were positive for Typhidot IgM, no patient $(0 \%)$ was positive for Typhidot IgG, while 12 (20\%) were negative for both Typhidot IgG and IgM. Amongst patients having fever for > 14 days, 4 (6.66\%) were positive for both Typhidot IgG and

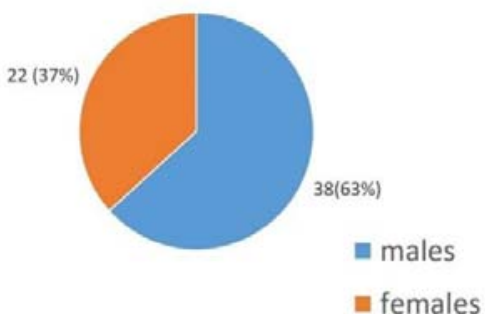

a

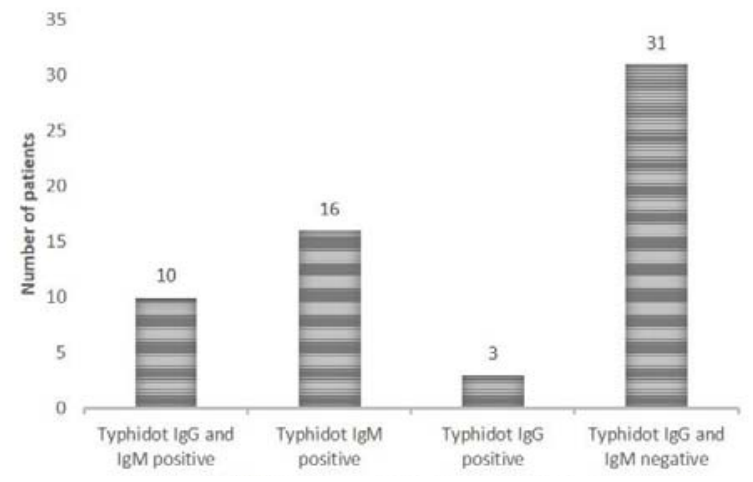

b

Figure 1: a: Gender distribution of paediatric patients, b: Results of Typhidot lgG and IgM for patients suspected of having typoid fever $(\boldsymbol{n}=60)$
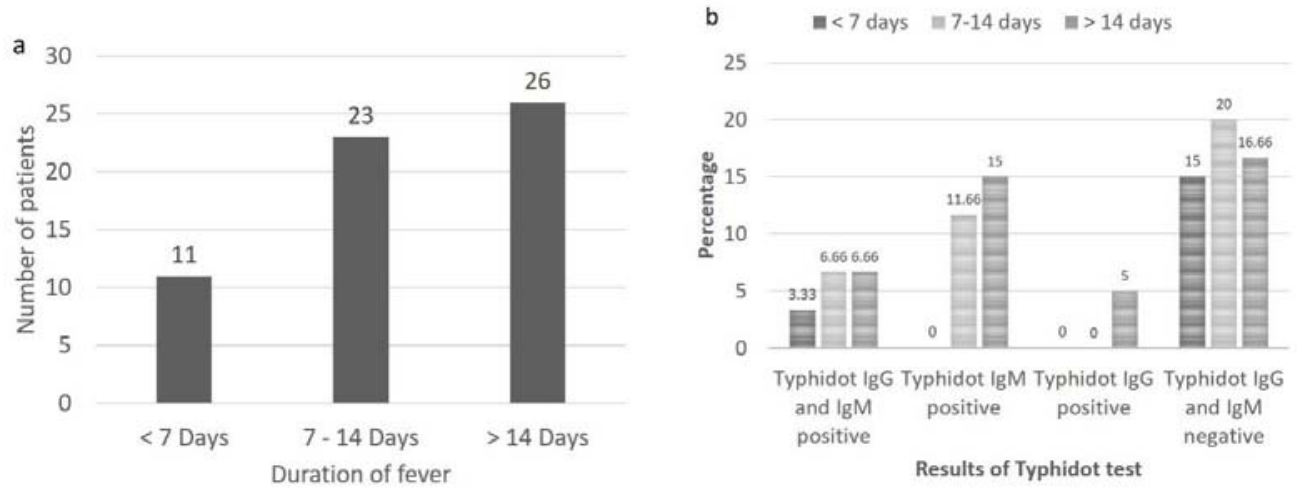

Figure 2: a: Duration of fever in patients suspected of having typhoid fever $(n=60)$, b: Results of Typhidot lgG and lgM according to duration of fever in patients suspected of having typhoid fever. 
IgM, 9 (15\%) were positive for Typhidot IgM, 3 (5\%) were positive for Typhidot IgG and $10(16.66 \%)$ were negative for both Typhidot IgG and IgM (Figure 2b).

In the present study, serological diagnosis of typhoid fever was also made using Widal TO and TH antigens. The results show that, of the total patients $(n=60), 8(13.33 \%)$ were positive for Widal TO and TH antigens, 3 (5\%) were positive for Widal T0 antigen, 19 (13.66\%) were positive for Widal TH antigen and 30 (50\%) were negative for Widal TO and $\mathrm{TH}$ antigens (Figure $3 \mathrm{a}$ ).

Out of 8 patients positive for Widal TO and TH antigens, equal number of males and females was affected. In 3 patients positive for Widal TO antigen all (100\%) were males and amongst 19 patients positive for Widal TH antigen 10 (52.63\%) were males and 9 (47.36\%) were females. Among rest of the $30 \mathrm{Widal} \mathrm{TO}$ and $\mathrm{TH}$ antigen negative patients 23 (76.66\%) were males and 7 (23.33\%) were females. Amongst patients having fever for $<7$ days, 1 (1.66\%) was positive for Widal TO and TH antigens, no patient $(0 \%)$ was positive for Widal TO antigen, 2 (3.33\%) were positive for Widal TH antigen and 8 (13.33\%) were negative for Widal TO and TH antigens. In patients having fever from 7-14 days, 1 (1.66\%) was positive for Widal TO and TH antigens, 2 (3.33\%) were positive for Widal TO antigen, 5 (8.33\%) were positive for Widal TH antigen and 15 (25\%) were negative for Widal TO and $\mathrm{TH}$ antigens. In patients having fever for $>14$ days, 6
(10\%) were positive for Widal TO and TH antigen, 1 (1.66\%) was positive for Widal T0 antigen, 12 (20\%) were positive for Widal TH antigen and 7 (11.66\%) were negative for Widal TO and TH antigens (Figure $3 \mathrm{~b}$ ).

In the present study, the clinical diagnosis was made according to set of symptoms and findings at physical examination. The common clinical presentation in patients suspected of having typhoid fever $(n=60)$ were fever 60 (100\%), abdominal pain 45 (75\%), nausea $38(63.33 \%)$, vomiting 40 (66.66\%), diarrhea 35 (58.33\%), constipation 15 (25\%), cough 40 (66.66\%), chest discomfort 34 (56.66\%), sore throat $37(61.66 \%)$, coated tongue 42 (70\%), anorexia $54(90 \%)$ and weakness 58 (96.66\%) (Table 1).

\section{Discussion}

Typhoid is considered as an illness of past by people who are residents of developed countries. It is a systemic, mortal and contagious disease caused by Salmonella typhi. This bacterium specifically affects humans in a highly-adapted manner. It is found more habitually in underdeveloped countries where overload of population and improper sanitation are common [15].

Molecular Immunology has made a headway to the discovery of sensitive and specific markers of Salmonella typhi. They are used to make such kits which are economical and factual for speedy serological interpretation of typhoid
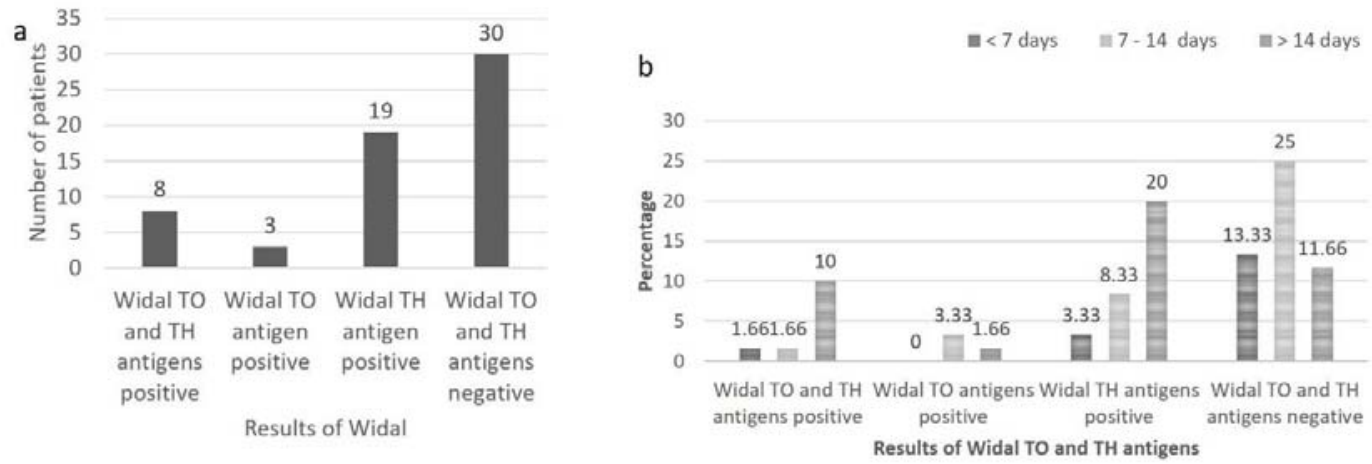

Figure 3: a: Results of Widal TO and TH antigen in patients suspected of having typhoid fever $(\boldsymbol{n}=60)$, b: Results of Widal TO and TH antigen according to duration of fever in patients suspected of having typhoid fever $(\boldsymbol{n}=60)$.

Table 1: Common clinical presentations in patients suspected of having typhoid fever $(n=60)$

\begin{tabular}{|c|c|c|}
\hline Clinical presentations & Patients with clinical presentations & Patients without clinical presentations \\
\hline Fever & $60(100 \%)$ & $0(0 \%)$ \\
\hline Abdominal pain & $45(75 \%)$ & $15(25 \%)$ \\
\hline Nausea & $38(63.33 \%)$ & $22(36.66 \%)$ \\
\hline Vomiting & $40(66.66 \%)$ & $20(33.33 \%)$ \\
\hline Diarrhea & $35(58.33 \%)$ & $25(41.66 \%)$ \\
\hline Constipation & $15(25 \%)$ & $45(75 \%)$ \\
\hline Cough & $40(66.66 \%)$ & $20(33.33 \%)$ \\
\hline Chest discomfort & $34(56.66 \%)$ & $26(43.33 \%)$ \\
\hline Sore throat & $37(61.66 \%)$ & $23(38.33 \%)$ \\
\hline Coated tongue & $42(70 \%)$ & $6000 \%)$ \\
\hline Anorexia & $54(100 \%)$ & $18(30 \%)$ \\
\hline Weakness & $58(96.66 \%)$ & $60(100 \%)$ \\
\hline
\end{tabular}


fever [16]. The serotype Typhi has a specific outer membrane protein (OMP) which is of $50 \mathrm{kDa}$. It was detected by Ismail, et al. who also checked out this antigen by dot-enzyme immunoassay (EIA) technique and noticed that this antigen can react with immunoglobulins $M$ and $G$ produced due to Salmonella typhi in the serum of the victims of acute typhoid fever [17]. The OMP is evident from the other antigens of the bacterium which are somatic $(0)$, flagellar $(\mathrm{H})$ or capsular (Vi). Interpreting from the current writings Typhidot is the most suitable test for confirmation of typhoid fever. Typhidot IgM positive means early phase of illness while both IgM and IgG are positive in case of middle phase of infection. IgG antibodies are not indicative of acute infection as they persist in serum of the patient for about 2 years of infection [12]. The results of Typhidot may vary topographically because of the distinctions in the genomic structure and flexibility of the outer membrane protein of Salmonella typhi [18]. While comparing the results of Typhidot (as given in figure 1b), another study including 65 patients showed positive results for both IgG and IgM in 39 samples, IgM was positive in 24 samples and IgG in 2 samples. Hence reporting that Typhidot is $100 \%$ sensitive and $80 \%$ specific as compared to blood culture [19].

The present study showed a notable pattern when the results were arranged according to the duration of fever (Figures 2a,b and $3 b$ ). It is comparable to the findings by Jaffery, et al. which reported that amongst the patients having fever for $<7$ days $(n=22), 10(55.56 \%)$ were positive for Widal and 12 (66.67\%) were positive for Typhidot. In cases with 7-14 days of illness ( $n=83)$, 38 were positive for Widal and 45 (80.36\%) were positive for Typhidot. Whereas in patients having illness of $>14$ days duration $(n=44), 24$ $(82.76 \%)$ were positive for Widal and $20(68.97 \%)$ were positive for Typhidot. The Typhidot showed an upper value of sensitivity and specificity to that of Widal test [16]. In another evaluation, the results of Typhidot showed high sensitivity during the first seven days of febrile illness. Seemingly, the reason for this is that while performing Typhidot, we are mainly concerned with the results of IgM which is the first antibody to be released after any infection. The level of IgG will be detectable later. In the Widal test, 0 agglutinins are observed at around day 8, while, $\mathrm{H}$ agglutinins are observed at about days 10-12 [20].

Underdeveloped countries have been using Widal test for more than hundred years but its advantage as a diagnostic tool is restricted because of less sensitivity, specificity and positive predictive value [21]. Sensitivity is reduced because Widal test may be positive after a long latent period. Specificity is reduced if the infection is repeated or patient has administered TAB vaccine or the ability to react with the other gran negative infections. When the blood culture test is combined with any serological test then it usually gives better diagnostic results specifically in those cases which are partially treated. This behavior could be tested by combining Typhidot with blood culture of those children who have already received antibiotics [22]. Routinely recommended Widal test usually does not give positive results in the first week of infection as it is dependent on increased titres which may take about 48 hours. The gold standard for typhoid is culture which is $100 \%$ specific and detect the antibiotic sensitivity. Its disadvantage is again a delay in the interpretation of results i.e. 72 hours. The results of culture are badly affected if there is prior administration of antibiotics. To overcome these drawbacks, serological tests like Typhidot and Tubex have been discovered in the recent past. These tests are helpful in the early diagnosis of typhoid fever by identifying antibodies against Salmonella typhi. Typhoid fever can be diagnosed within 20 minutes by using Typhidot test. The usefulness of Typhidot is quite profound in the diagnosis of typhoid fever. It is easy to perform, and results are interpreted quickly [12]. There is no need of an ELISA reader to evaluate the results. They are easily reported by seeing the dots on the test strips. It can easily be performed without much operator training. The hindrances in advising this test in developing countries are the cost of the test which is more than Widal test and the test reagents must be placed in cold-storage [22].

\section{Conclusion}

For the diagnosis of typhoid fever, Typhidot is much better than Widal test as it is more sensitive, easy to perform, detects the cases at early stage and results are available on the same day. And in Typhidot test, IgM is more prevalent than IgG meaning that more are the cases of acute infection as compared to chronic infection.

\section{Ethical approval}

Ethical approval was taken from Children's hospital. An informed consent was taken from all the subjects involved.

\section{Acknowledgement}

We acknowledge The University of the Punjab and Children's hospital management for their help in this research work.

\section{References}

1. Crump JA, Sjölund KM, Gordon MA, Parry CM. Epidemiology, clinical presentation, laboratory diagnosis, antimicrobial resistance, and antimicrobial management of invasive Salmonella infections. Clin Microbiol Rev. 2015; 28: 901-937.

PubMed: https://www.ncbi.nlm.nih.gov/pubmed/26180063

2. De Jong HK, Achouiti A, Koh GC, Parry CM, et al. Expression and function of S100A8/A9 (calprotectin) in human typhoid fever and the murine Salmonella model. PLoS Negl Trop Dis. 2015; 9: e0003663. PubMed: https://www.ncbi.nlm.nih.gov/pubmed/25860480

3. WHO. Typhoid: Immunization, Vaccines and Biologicals. 2015.

4. Steele AD, Hay Burgess DC, Diaz Z, Carey ME, Zaidi AK. Challenges and opportunities for typhoid fever control: a call for coordinated 
action. Clini Infect Dis. 2016; 62: S4-S8.

PubMed: https://www.ncbi.nlm.nih.gov/pubmed/26933019

5. Ayub U, Khattak AA, Saleem A, Javed F, Siddiqui N, et al. Incidence of typhoid fever in Islamabad, Pakistan. Am-Eurasian J Toxicol Sci. 2015; 7 : 220-223.

6. Akullian A, Ng'eno E, Matheson Al, Cosmas L, Macharia D, et al. Environmental transmission of typhoid fever in an urban slum. PLoS Negl Trop Dis. 2015; 9: e0004212.

PubMed: https://www.ncbi.nlm.nih.gov/pubmed/26633656

7. Mehmood K, Sundus A, Naqvi IH, Ibrahim MF, Siddique $O$, et al. Typhidot-A blessing or a menace. Pak J Med Sci. 2015; 31: 439-443. PubMed: https://www.ncbi.nlm.nih.gov/pubmed/26101507

8. Kumar KS, Suganya M, Sathyamurthi B, Anandan H. Reliability of Typhidot Rapid Immunoglobulin M and Immunoglobulin $G$ in the diagnosis of Typhoid fever. Int J Sci Study. 2016; 4: 256-259.

9. Khan MN, Shafee M, Hussain K, Samad A, Awan MA, et al. Typhoid fever in paediatric patients in Quetta, Balochistan, Pakistan. Pak J Med Sci. 2013; 29: 929-932.

PubMed: https://www.ncbi.nlm.nih.gov/pubmed/24353661

10. Prasad K, Oberoi J, Goel N, Wattal C. Comparative evaluation of two rapid Salmonella-lgM tests and blood culture in the diagnosis of enteric fever. Indian J Med Microbiol. 2015; 33: 237-242.

PubMed: https://www.ncbi.nlm.nih.gov/pubmed/25865974/

11. Krishna S, Desai S, Anjana V, Paranthaaman R. Typhidot (IgM) as a reliable and rapid diagnostic test for typhoid fever. Annals Tropi Med Public Health. 2011; 4: 42.

12. Muthaiyan J, Moomin MRA, Varadarajan P, Jayachandran G. A comparative study of Typhidot test and Widal test with the culturepositive typhoid fever in children. Cough. 2016; 15: 57.

13. Keddy KH, Sooka A, Letsoalo ME, Hoyland G, Chaignat CL, et al. Sensitivity and specificity of typhoid fever rapid antibody tests for laboratory diagnosis at two sub-Saharan African sites. Bull World
Health Organ. 2011; 89: 640-647.

PubMed: https://www.ncbi.nlm.nih.gov/pubmed/21897484

14. Jindal N, Bansal R, Grover P, Malhotra R, Singh S. Rapid diagnosis of typhoid fever-a comparative study of typhidot and widal test. Int J Bioassays. 2014; 3: 3438-3440.

15. Mitra R, Kumar N, Trigunayat A, Bhan S. New advances in the rapid diagnosis of typhoid fever. African J Microbiol Res. 2010; 4:17471753.

16. Jaffery G, Anwar SM, Hussain W, Maqbool S. Serological diagnosis of typhoid fever in children: a comparative evaluation of Salmonella typhi O-9 antigen based rapid essay. 2005.

17. Buigut JA. Evaluation of widal tube dilution and enzyme-linked immunosorbent assay for diagnosing typhoid fever at a referral hospital in Kenya. Moi University. 2015.

18. Choo K, Davis T, Ismail A, Ibrahim TT, Ghazali W. Rapid and reliable serological diagnosis of enteric fever: comparative sensitivity and specificity of Typhidot and Typhidot-M tests in febrile Malaysian children. Acta Trop. 1999; 72:175-183.

PubMed: https://www.ncbi.nlm.nih.gov/pubmed/10206117

19. Jesudason M, Esther E, Mathai E. Typhidot test to detect IgG \& IgM antibodies in typhoid fever. Indian J Med Res. 2002; 116: 70-72. PubMed: https://www.ncbi.nlm.nih.gov/pubmed/12592993

20. Olsen SJ, Pruckler J, Bibb W, Thanh NTM, Trinh TM, et al. Evaluation of rapid diagnostic tests for typhoid fever. J Clini Microbiol. 2004; 42:1885-1889.

PubMed: https://www.ncbi.nlm.nih.gov/pubmed/15131144

21. Sherwal B, Dhamija R, Randhawa V, Jais M, Kaintura A, et al. A comparative study of Typhidot $M$ and widal test in patients of Typhoid fever. J Indian Academy Clini Med. 2004; 5.

22. Yadav K, Yadav SK, Parihar G. A comparative study of typhidot and Widal test for rapid diagnosis of Typhoid fever. Int $\mathrm{J}$ Curr Microbiol App Sci. 2015; 4: 34-38. 